\title{
BEAM DYNAMICS ISSUES in LINEAR COLLIDERS*
}

\author{
John T. Seeman \\ Stanford Linear Accelerator Center \\ Stanford Universitv. Stanford. CA 94309
}

\begin{abstract}
The primary goal of present and future linear colliders is to maximize the integrated luminosity for the experimental program. Beam dynamics plays a central role in the maximization of integrated luminosity. It is the major issue in the production of small beam sizes and low experimental backgrounds and is also an important factor in the production of particle numbers, in the acceleration process, and in the number of bunches. The bcam dynamics effects on bunches which are extracted from the damping rings, accelerated in the linac, collimated, momentum analyzed, and finally delivered to the final focus are reviewed. The effects of bunch compression, transverse and longitudinal wakefields, BNS damping, energy definition, dispersion, emittance, bunch aspect ratio, feedback, and stability are all important.
\end{abstract}

\section{Introduction}

Active investigations of the effects of beam dynamics on linear colliders have been pursued for about a decade $[1,2,3]$. This work has been carried out in many laboratories around the world, including institutions such as CERN, Cornell, DESY, Frascati, INP, KEK, Orsay, SAI, SLAC, UCLA, and others. Significant progress has becn made on the theoretical understanding of the various effects. Experimental confirmations are now starting to appear coming from the first linear collider, the SLC at SLAC $[4,5,6]$, and from other laboratories. Many new constraints have been discovered which must be incorporated in the design of future colliders

There are many beam dynamics issucs. A list of many of them is shown in Table $I$. As a full discussion, complete with equations and derivations, would require far more space than available, the issues will be described only in terms of their physics aspects, their motivation, and interdependence. Issues concerning the final focus are mostly unique to that region and are covered in other papers in this conference.

The cross-coupling among the various beam dynamics effects is very strong. Here is one example. The longitudinal length of each bunch affects the energy and energy spectrum. The length and energy spectrum of each bunch affect the emiltance enlargement from current dependent effects. The emittance enlargement contributes to the minimum beam diameter at the final focus and to the detector backgrounds. Many other interdependencies exist.

\section{Linear Collider Goals}

The beam parameters which are used to generate luminosity for physics tie directly to the phase space volume of the beam as it is accelerated. The bunches enter the system with an energy of one to three $\mathrm{GeV}$ and exit with energies of 50 to $1000 \mathrm{GeV}$ depending on the design. The RF phase of the linac is adjusted to produce energy spectra of about 0.2 to 0.5 percent. The bunch length is shortened so that transverse wakefields do not enlarge the emittance but not too short as to produce excessive longitudinal beam loading. Bunch lengths of 50 to 3000 microns are required. The transverse beam sizes are on the order of 5 to 120 microns (sigma) in the accelerator. The invariant emittances of the bunches $\left(3 \times 10^{* *}-7 \mathrm{r}-\mathrm{m}\right.$ to $3 \times 10^{* *}-5 \mathrm{r}-\mathrm{m}$ ) push the frontiers of damping rings and transport systems [7]. In order to obtain maximal luminosity as many bunches as possible must be accelerated which introduces multibunch RF loading, long range wakefields, and instrumentation problems. $A$ beam dynamicist must satisfy all these requirements when operating or designing a collider.

\section{Bunch Length Preparation}

The relatively long length of a bunch in a damping ring (6 to $10 \mathrm{~mm}$ ) must be shortened by one to two orders of magnitude before it can be accelerated in the main collider linac. This task is perfomed by a non-isochronous bend in conjunction with an RF 'compressor'. The bunch passes through the compressor at the zero amplitude phase such that the head of the bunch is accelerated and the tail is decelerated. If the amplitude of the $R F$ is adjusted properly, the bunch head, tail, and center arrive at the linac entrance at the same time. The bunch length is then determined by the energy spectrum of the beam leaving the damping ring. A longer bunch can be produced by using a lower RF amplitude.

The beam in the non-isochronous bend is quite large transverscly in order to provide path length differences. As a result, the first and second order optics must be carefully analyzed to reduce emittance enlargements. Some of the possible errors arc residual dispersion (energy-position correlations), betatron mismatches, horizontal-vertical coupling, transverse RF deflections, and non-gaussian transverse and longitudinal density distributions.

There are possibilitics of shaping the longitudinal profile of the bunch by careful collimation at selected positions in the non-isochronous bend. Shaping has the potential of reducing the strength of transverse wakefields in the linac. This possibility has not been fully studied.

In order to produce lengths on the order of 50 microns the bunch must pass through two compression regions with significant (10 to $20 \mathrm{GeV}$ ) acceleration between. This multiple compression provides both the opportunity to reduce errors of a single system and the possibility of generating more complicated correlated errors. A complete analysis of this double compression scheme is presently under analysis.

When electron polarization is used with a linear collider the bunch compression becomes more complicated as spin rotating solenoids must be used. The solenoids have strong focusing and coupling properties. When flat beams are desired as well, spin rotators force the use of additional skcw quadrupoles and spccial betatron matching. 
Table I List of Beam Dynamics Issues

I. Bunch length preparation:

a) 1 st and 2nd order optics

b) Non-gaussian distributions

c) Possible length shaping

d) Correlated crrors during

multiple compressions

e) Spin rotators

II. Trajectory correction:

a) Correction algorithms

b) Quadrupole misalignments

c) Bcam position monitor offsets

d) Multiple bunches

e) Long range phase advance errors

f) Failed components

III. Detector backgrounds:

a) Tail particles

b) Collimator placement

c) Collimator beam heating

d) Collinator beam steering

e) Beta mismatch changes

with encrgy changes

f) Transverse beam dimensions

IV. Emittance enlargement from single particle effects:

a) Beta mismatch (initial)

b) Beta mismatch (quad errors)

c) Beta mismatch (energy errors)

d) Driven betatron mismatch

e) Dispersion mismatch (initial)

f) Dispersion mismatch from

quad and BPM misalignments

\section{Trajectory Correction}

The bcam must have a trajcctory near the center of the irises of the RF structure in order to avoid transverse wakeficld effects and near the magnetic center of the quadrupoles to avaid chromatic emittance enlargement. The trajectory correction algorithm can take many forms including rms reduction, closed bumps, one-to-one steering, two beam steering, harmonic reduction, and others. For a system without broken hardware and infinitely strong correction dipoles, most algorithms produce identical results. However, with missing monitors (a few will always be broken) and finite strength correctors, different algorithms produce different final trajectories. The key is exception handling. The errors remaining in the final trajectory determines which dynamics issue dominates. If coherent trajectories remain, systematic emittance blowup occurs. If random errors remain, stochastic enlargement occurs. Often both appear,

With multiple bunches in each RF pulse, the correction of each bunch may be impossible and an averaging process must be used. This will tighten tolerances.

Errors in the transverse placement of quadrupoles and errors in the effective centroid of the position monitors will affect the trajectory correction. The result of these errors is a stochastic process. These offset errors can be studied using the beam and corrected.

Trajectory correction over many betation wavelengths can bc difficuls as small quadrupole strength errors rclative to the local beam energy can cause large betatron phase errors over the entire machine. The g) RF deflections

h) Multipole fields

i) Filamentation rate

j) Multiplicative effects

h) Additive effects

i) $\quad X-Y$ coupling

V. Emittance enlargement from current dependent effects:

a) Transverse wakeficlds

1) Position jitter tolerance

2) BNS damping (E spectrum)

3) BNS damping (RF focusing)

4) Lattice choice

5) Injection dispersion

6) Tail generation

7) Core distortion

8) Head-Tail offsets

9) Collimator deffections

b) Trajectory jitter

1) Accelcrator vibration
2) Quadrupole vibration
3) Quadrupole strength jitter
4) Dipole amplitude jitter
5) RF deflection jitter from
amplitude and phase

VI. Encrgy and energy spectrum:

a) Longitudinal wakefields
b) RF acceleration
c) Bunch length
d) Muliple bunches
e) KIystron phase jitter
f) Klystron amplitude jitter
g) Overall RF phase
h) BNS damping
i) Energy feedback overhead
j) Accelerator temperature stability
k) Momentum collimation

number of oscillations in the various linacs that have been discusscd ranges from 30 to 1000 . This allows only small strength errors before oscillations can not be predicted over a long range. Separating the linac into multiple correction regions improves the convergence but increases the trajectory correction time.

\section{Detector Backgrounds}

Detector backgrounds which interfere with physics data collection can come from many sources. Particles at large transverse amplitudes can strike the vacuum chamber near the detector and produce shower debris. Muons can be generated upstream and are difficult to remove or deflect. The large beam sizes in the strong final focus quadrupoles produce synchrotron radiation which is hard to mask. Backgrounds from beam-beam effects are also expected. Many of the beam dynamics issues discussed bclow contribute to particles at large amplitudes.

Collimation is not easy. The placement of collimators must be carefully planned as intense beams near collimator jaws can be deflected with angles comparable to their natural divergence. Small intense beams can crack collimator jaws on single strikes. The power in the beam is large and average power limits for collimators can easily bc exceeded. A substantial intensity loss of a beam on a collimator will change how that beam is matched to the transport lattice.

Mismatches in the beam shape with respect to that expected from the natural quadrupole lattice can lead to 
background sensitivities to machine changes. Collimation is sensitive to changes in the betatron phase advance, energy gain profiles, beam shape in collimators, and transverse tail profile. Thus, collimator placement must dictate some conditions on the overall layout of the accelerator.

\section{Emittance Enlargement from Single Particle Effects}

Single particle dynamics determine the first and second order properties of the beam transport from the damping ring to the final focus. The design process must incorporate all of these effects as they determine the baseline emittance on which current dependent effects add

\section{Betatron filamentation}

If the betatron phasc space is mismatched to the accelerator transport lattice, the phase space will filament due to the finite energy spectrum in the beam. Several causes for betatron mismatches are errors in the damping ring lattice, the length compression process, the linac quadrupoles, or the energy gain profile. The energy spectrum varies depending on BNS damping and on the distance along the linac. The filamentation for betatron mismatches will occur at twice the rate as for coherent betatron oscillations because of symmetry. This process increases the emittance of the beam but maintains the transverse gaussian profile. The emittance growth is quadratic in the mismatch for small errors but becomes linear with large offsets. In practice, matching the betatron phase space to the lattice is expensive as elaborate equipment is needed and substantial beam time is required Furthermore, unraveling the causes of filamentation after the fact is difficult as several unrelated causes can contribute.

A beam that is launched offaxis or is given a transverse deflection will execute a betatron oscillation. Because of these oscillations the phase space will filament. The extent of filamentation depends linearly on the energy spectrum in the bunch and the number of betatron oscillations. A fully filamented bunch will have a shape which matches the quadrupole lattice but will not have a gaussian profile. The emittance will be larger. A partially filamented bunch will have a centroid position that is offaxis. Its effective betatron function will not match the quadrupole lattice and the effective emittance will lie between the initial and the fully filamented valuc. The shape of a partially filamented bunch will tend to change with time as the locations and amplitudes of the deflections will change

The allowed deflections will depend on the betatron function and the beam energy at the location of the deflection. The combined effect of the deflections are added statistically if they are random and linearly of they are systematic. The combined effects are propagated downstream. The tolerance level is set by the emittance of the beam and the allotted growth

\section{Coupling}

Coupling of the horizontal and vertical betatron motion via skew fields can cause an actual or an apparent emitlance growth. This places tolerances on quadrupole rotations and trajectories in sextupoles. This constraint is usually not difficult to satisfy except for colliders with very flat beams.

Dispersion

Energy position correlations (dispersion) will enlarge the emittance if allowed to filament or is uncompensated. Dispersion enters the beam either by unintentional leaking of dispersion from the bunch length compression section, through 'trajectory bumps', or by free betatron oscillations. The trajectory bumps are local and are caused by beam position monitor errors or quadrupole offsets. The trajectory correction algorithm generates these local bumps as as a matter of course. The bumps are unobserved in a trajectory measurement because the trajectory appears to be centered.

Filamentation from dispersion is different from that of betatron mismatches because the particle position offsets depend on the local energy spectrum which changes rapidly along the linac. For example, an anomalous dispersion which exits the compression section will produce transverse offsets determined by the incoherent energy spectrum at that point. However, the incoherent spectrum, which does not depend on longitudinal position, is very rapidly changed along the linac to a coherent spectrum, which does depend on longitudinal position. The coherent spectrum is determined by BNS damping. The amplitude of the particle offsets do not change rapidly leading to direct emittance growth.

\section{RF Deflections}

RF deflections also cause emittance growth. The RF structure is designed to provide longitudinal acceleration. Due to small errors in construction, the fields are rotated a small amount and transverse acceleration or deflection occurs. The ratio of transverse to longitudinal acceleration is not more than $1 / 1000$ and is difficult to measure. Observations of these deflecting fields show that they need not be in phase with the longitudinal fields. A 45 degree phase difference is not uncommon. Since the beam has a finite bunch length, the deflection will vary for particles at different positions along its length. A nearby dipole will remove the net deflection during the course of trajectory correction, leaving opposite deflections for the head and tail. This is a direct emittance enlargement or an anomalous dispersion if there is a head-tail energy correlation. This form of phase space dilution grows with the random deflections along the linac. The most sensitive location for RF deflections is the compressor accelerator near the damping ring where the bunch length is relatively long. Its deflections appear as anomalous dispersion.

\section{Multipole fields}

Multipole fields in general are unimportant for linear collider linacs as the beams are so small. However the bunch length compression sections must have second order optics corrections and need multipole magnets because of the large beams sizes and the large energy spectrum. Downstream in the linac the beams are dense and very energetic. A beam strike on a linac iris caused by a component malfunction upstream would damage the RF structure on a single pulse. It is possible to protect the irises by using multipoles to expand the beam as it deviates far from the axis. Finally, collimation is difficult with metallic surfaces and a multipole collimation system may be a good compromise.

The various single particle effects discussed above add to the natural emittance of the beam in different ways. Betatron mismatches after filamentation multiply the initial natural beam emittance. Dispersion and filamentation due to oscillations produce increases in the emittance which add in quadrature.

\section{Current Dependent Emittance Enlargement}

Transverse wakefields produced by a bunch traveling off-axis in an RF structure change the dynamics of the individual particles in that bunch. In order to make maximum luminosity the beam intensities are increased until these effects produce emittance enlargements 
comparable to other effects. Ultimately, all linear colliders will be limited by these effects. A bunch can pass off-axis through an accelerating structure from betatron oscillations, local bumps, head-rail transverse offsets, or collimator deflections. The results of these perturbations vary.

Sources of off-axis bunches:

A bunch will exhibit a betatron oscillation if launched anywhere along the transport system with an offset in pusition or angle from the accelerator axis. Static launch errors can originate from several sources: damping ring extraction kickers, dipole correction magnets, RF deflections, transverse shifts of quadrupoles, or setting changes of an off-axis quadrupole. Static errors in launch can be corrected using standard trajectory correction methods, except that the correction is more difficult when wakefields change the observed betatron phase advance from what is expected. Trajectory correction which divides the linac into sections tends to capturc local betatron oscillations of finite length because the beam moves between different steps of the correction process. This results in 'frozen oscillations' in the lattice. The consequences of a betatron oscillation depend on the number of oscillations, the local lattice, the energy of the beam, and the strength of the wakefields. The best quadrupole lattices to reduce wakefield effects have phase advances of around 100 degrees per cell. Time dependent trajectory changes are more difficult to deal with. Feedback systems can be devised to reduce errors which occur slower than about one sixth the machine repetition rate if beam feedback is used. Very fast trajectory jitter can only be reduced by sensing the actual changing component or by minimizing its impact. Time dependent jitter typically comes from kicker jitter, accelerator vibration, quadrupole vibration, dipole or quadrupole power supply variations, RF amplitude and phase changes coupled with RF deflections, or feedback chatter.

Local trajectory errors can also occur. A mis-aligned accelerator section generates local wakeficld effects which do not change the head of the bunch but drives the core and the tail into oscillations. The core oscillation then drives the wakefield enlargement of the tail throughout the remainder of the accelerator. A second kind of beam displaccment occurs when quadrupoles or position monitors have offsets. In this case the trajectory correction program makes local trajectory bumps to correct the error. These local bumps cause wakefield effects which add statistically along the linac.

Head-tail transverse offsets of the bunch about the accelerator axis will drive wakefield emittance dilution unless local cancellation can be achieved. Thesc offsets are such that the longitudinal head of the bunch is on one side of the axis and the tail on the other. The core is centered. These offsets can not be corrected by simple dipole corrections. Some of the possible causes are RF deflections from either the compressor accelerator or the main accelerator, angle misalignments of accelerator sections, anomalous dispersion exiting the compression region in conjunction with non-minimum bunch lengths, and chromatic steering effects from misaligned components in conjunction with head-tail energy differences. Cancellation can be obtained by using special stcering in the accelerator if the errors are known. The consequence of these head-tail offsets is that a finite emittance increase is unavoidable.

Bunched beams passing very close to metallic surfaces such as a collimator will be transversely deflected at asymmetric transitions. This effect causes the core of the beam to be deflected more than the tails which directly increases the emittance and drives wakeficlds downstream. For linear collider beams of very low emittance this effect can be severe.
Effects of transverse wakefield:

Transverse wakefields produce emittance enlargement. Off-axis particles generate wakefields in the accelerating structure which deflect all trailing particles. The deflections accumulate coherently (for example for free betatron oscillations) or incoherently (for example for results of quadrupole offsets). The growth is very rapid for bunches which have the same oscillation frequency for all particles. A typical case is a free betatron oscillation. The bunch is launched off-axis. The head of the bunch drives the core and tail to ever increasing amplitudes. The position of the core eventually has a 90 degrees phase lag from the head as deflections must turn into positions. The tail of the beam eventually is driven hardest by the core which has most of the charge of the bunch and a large position offset and develops a 90 degree phase lag from it. The head-tail phase lag is about 180 degrees. Of course. a careful integration is needed to determine the exact phase space position of each particle. Tolerances on the size of the above mentioned accelerator features can be computed with a simulation program constrained by an acceptable emittance enlargement.

Reduction of transverse wakefields:

The resonant enlargement of beams by wakefields can be reduced by a scheme called BNS damping named after Balakin, Novokhatsky, and Sminnov from Novosibirsk [8]. Their effect is to reduce the effective defocusing nature of the wakefield force by providing extra focusing for the core and tail particles. This is accomplished by lowering the energy of the trailing particles relative to the head so that the quadrupole lattice focuses them more strongly. The tail particles are lowered in energy by backphasing klystrons early in the accelerator and forwardphasing downstream klystron to keep the energy spectrum small at the maximum energy. The best configuration depends on many machine parameters and must be calculated for each case. Tests of BNS damping at the SLC collider have been very successful [9] and BNS damping is used routinely. Another form of wakefield compensation using a similar scheme was invented at CERN where RF focusing is used to provide the extra focusing for the core and tail of the beam [10]. Some form of BNS damping has been incorporated in all future collider designs.

If multiple bunches are used in the collider to increase the luminosity, long range wakefields must be carefully reduced. The reduction can be obtained (1) by removing the higher mode power left by earlier bunches, (2) by making each cell different so that the higher order modes decohere, or (3) by maximizing the distance between bunches.

\section{Energy and Energy Spectrum}

Longitudinal wakefields affect the acceleration and energy spectrum of the beam. At low currents the bunch rides on the crest of the RF sine wave and has a small energy spectrum ( about $0.2 \%$ ) for short bunch lengths. At high currents the longitudinal wakefields depress the energy of the tail. The overall RF phase must be advanced so that the bunch rides ahead of the crest where the curvature of the sine wave cancels 10 first order the longitudinal wake. The shorter the bunch the stronger the longitudinal wakefields are. The longer the bunch the stronger the transverse wakefields. A compromise must always be reached.

There are several contributions to the required RF acceleration. The particles must be accelerated to the desired energy. Advancing the phase to compensate the 
longitudinal wakefields also costs RF voltage. For example. in the SLC at $6 \times 10^{* * 10}$ particles per bunch the accelerator must provide about 10 percent more integrated gradient than the output energy would indicate. Furthermore, BNS damping with phases adjusted to provide a coherent energy spectrum along the linac costs additional integrated gradient. Any overhead required for the energy feedback system must be provided. The phases of individual klystrons and of the subboosters drift slowly and some additional RF voltage is needed for compensation. Finally, the temperature of the accelerating structure must be kept constant so that phase errors do not accumulate inside the structure.

Multiple bunch acceleration poses the problem of keeping the energy of all the bunches cqual and their energy spectra small. By staggering in time the RF filling of the accelerator sections most of these problems can be solved but more RF voltage is again needed.

\section{Conclusions}

In this note Inany issues of beam dynamics have been discussed. Most of them do not affect a given collider design. Nevertheless, each of these effects surfaces in one design or another. Care must be taken to look at them all before determining the more important points.

The topics of intense interest at the moment [11] are multiple bunch operation, injection mismatches (dispersion, betatron functions), transverse wakefield reduction, $x-y$ coupling, collimation, and RF deflections.

\section{References}

1) U. Amaldi, "Colliding Linacs," CERN EP/79-136.

2) R. Stiening, "An Introduction to the Accelerator Physics of Lincar Colliders," AIP Conference Proceedings of the SLAC Summer School on Particle Accelerators, No, 105 , p. $281,1982$.
3) A. Skrinsky, "Linear Colliders," 12th Int. Conference on High Energy Accelerators, FNAL, 1983, p. 104.

4) J. Seeman and J. Sheppard, "Special SLC Linac Developments," 1986 Linear Accelerator Conference, SLAC, Stanford, CA, June 1986, p. 441.

5) J. Seeman, et al., 'Experimental Beam Dynamics in the SLC Linac," Particle Accelerator Conference, Washington D.C., March 1987, IEEE 87CH2387-9, p. 73.

6) J. Seeman, "Beam Dynamics Verification in Linacs of Linear Colliders," 1988 Linear Accelerator Conference, Williamsburg, Virginia, October, 1988.

7) R. Ruth, "Emittance Preservation in Linear Colliders," US/CERN Joint Topical Course on 'Frontiers in Particle Beams', South Padre Island, Texas, October, 1986.

8) V. Balakin, A. Novokhatsky, and V. Smimov, "VLEPP: Transverse Beam Dynamics," 12th Int. Conference on High Energy Accelerators, FNAL, 1983, p. 119.

9) J. Seeman et al., "First Observation of Transverse Wakefield Damping in a Linear Accelerator," SLAC report PUB-4968, (to be published), May 1989.

10) H. Henke, "Transport and Acceleration of LowEmittance Electron Beams," 1988 Linear Accelerator Conference, Williamsburg. Virginia. October, 1988.

11) Beam Dynamics Working Group (R. Ruth, Chairman), International Workshop on Next-Generation Linear Colliders, SLAC Report 335, December 1988. 